\title{
Crop/Forage/Soil Management/Grassland Utilization
}

\author{
Department of Crop and Soil Sciences, Cornell University, Ithaca, NY, USA
}

\section{Nitrogen, Phosphorus, Potassium, Magnesium and Calcium Removal by Brown Midrib Sorghum Sudangrass in the Northeastern USA}

\author{
Q. M. Ketterings, G. Godwin, T. F. Kilcer, P. Barney, M. Hunter, J. H. Cherney, and S. Beer
}

\begin{abstract}
Authors' addresses: Dr Quirine M. Ketterings (corresponding author; e-mail: qmk2@cornell.edu), Associate Professor, Department of Crop and Soil Sciences, Cornell University, 817 Bradfield Hall, Ithaca NY 14853; Mr G. Godwin, Research Support Specialist, Department of Crop and Soil Sciences, Cornell University, 813 Bradfield Hall, Ithaca NY 14853; Mr T. F. Kilcer, Field Crops Extension Educator, Cornell Cooperative Extension of Rensselaer County, 61 State St, Troy, NY 12180; Mr P. Barney, Field Crops Extension Educator, Cornell Cooperative Extension of St Lawrence County, 1894 State Highway 68, Canton, NY 13617; Mr M. Hunter, Field Crops Extension Educator, Cornell Cooperative Extension of Jefferson County, 203 North Hamilton Street, Watertown, NY 13601; Dr J. H. Cherney, Professor, Department of Crop and Soil Sciences, Cornell University, 513 Bradfield Hall, Ithaca NY 14853; Mr S. Beer, Research Support Specialist, Department of Crop and Soil Sciences, Cornell University, 505 Bradfield Hall, Ithaca NY 14853, USA
\end{abstract}

With 5 tables

Received January 21, 2006; accepted March 4, 2006

\begin{abstract}
For the long-term sustainability of the dairy industry in the Northeastern USA, manure nutrient application rates should not exceed crop nutrient removal once aboveoptimum soil fertility levels are reached. Dairy producers have shown a growing interest in brown midrib (BMR) forage sorghum (Sorghum bicolor (L.) Moench.) $\times$ sudangrass (Sorghum sudanense Piper) hybrids $(\mathrm{S} \times \mathrm{S})$ as a more environmentally sound alternative to maize (Zea mays L.) but data on $\mathrm{S} \times \mathrm{S}$ nutrient removal rates are scant. Our objectives were to determine $\mathrm{N}, \mathrm{P}, \mathrm{K}, \mathrm{Ca}$ and $\mathrm{Mg}$ removal with harvest as impacted by $\mathrm{N}$ application rate, using six $\mathrm{N}$ rate studies in New York. One of the six sites had a recent manure history. Although site-to-site differences existed, $\mathrm{N}$ application tended to decrease $\mathrm{P}$ and $\mathrm{K}$ and increase $\mathrm{N}, \mathrm{Ca}$ and $\mathrm{Mg}$ concentrations in BMR $\mathrm{S} \times \mathrm{S}$ forage. Nutrient removal and yield were highly correlated for all sites except one location that showed a $\mathrm{K}$ deficiency. The crop removed large amounts of $\mathrm{P}$ and $\mathrm{K}$ in the manured site, suggesting that $\mathrm{BMR} \mathrm{S} \times \mathrm{S}$ is an excellent scavenger of these nutrients. If manure is applied mid-season, forage $\mathrm{K}$ levels are likely too high for feeding to non-lactating cows.
\end{abstract}

Key words: brown midrib - macronutrients nitrogen — northeastern USA — nutrient removal — sorghum $\times$ sudangrass

\section{Introduction}

In the past 5 years, dairy producers in the northeastern USA have shown a growing interest in brown midrib (BMR) sorghum (Sorghum bicolor (L.) Moench.) $\times$ sudangrass (Sorghum sudanense Piper) hybrids $(\mathrm{S} \times \mathrm{S})$ as an annual forage crop managed in a two-cut system. On dairy farms, $\mathrm{S} \times \mathrm{S}$ has environmental benefits over maize (Zea mays L.), as it is planted in June (vs. the April/May planting window for maize) and allows for manure application following first cutting in July/August when field conditions are less conducive to runoff and/or leaching in the humid northeastern USA. However, for the long-term sustainability of the dairy industry in the region, manure nutrient application rates should not exceed crop removal of nutrients once an above-optimum agronomic fertility status is reached. This is especially relevant for nitrogen $(\mathrm{N})$ and phosphorus $(\mathrm{P})$, nutrients of environmental concern (U.S. Environmental Protection Agency 2000), and for potassium (K), a nutrient that, if present in excess, could cause animal health problems in non-lactating cows (Beede 1996, Goff and Horst 1997).

For many forage crops in North America, average forage nutrient concentrations are available through published summaries (e.g. PPI/PPIC/ FAR 2002), or electronic databases maintained by commercial forage laboratories (e.g. Dairy One 2006). However, data on $\mathrm{S} \times \mathrm{S}$ nutrient removal rates are scant. 
Nitrogen is often limiting dry matter (DM) production of $\mathrm{S} \times \mathrm{S}$ (Beyaert and Roy 2005) and $\mathrm{N}$ application may impact $\mathrm{P}, \mathrm{K}$, calcium $(\mathrm{Ca})$ and magnesium $(\mathrm{Mg})$ concentrations in the forage. In a 2-year field study conducted in Central NY, N application rate did not impact $\mathrm{K}$ levels of BMR $\mathrm{S} \times \mathrm{S}$, while $\mathrm{K}$ application increased forage $\mathrm{K}$ concentrations but did not impact DM yields (Ketterings et al. 2005a). Field trials conducted at seven locations in NY in 2002-2003 showed that $\mathrm{P}$ concentrations of BMR $\mathrm{S} \times \mathrm{S}$ were higher if $\mathrm{N}$ availability limited yields (Ketterings et al. 2004) and similar interactions are likely for $\mathrm{Mg}$ and $\mathrm{Ca}$. However, additional research is needed to quantify such nutrient interactions and to investigate the possibility of deriving crop nutrient removal rates from DM yields across multiple locations.

Our objectives were to determine (i) impact of $\mathrm{N}$ application on $\mathrm{N}, \mathrm{P}, \mathrm{K}, \mathrm{Ca}$ and $\mathrm{Mg}$ concentrations in a 2-cut forage BMR $\mathrm{S} \times \mathrm{S}$ system, and (ii) relationships between yield and nutrient removal with harvest across multiple locations.

\section{Materials and Methods \\ Locations}

Six N rate studies were conducted in 2004 in northern NY (Jefferson, St Lawrence, Essex Counties), eastern NY (Columbia County), and central NY (Cayuga and Tompkins Counties). Soils ranged from loamy fine sand in Essex County to silt loams in Jefferson and St Lawrence Counties (Table 1) and covered major agricultural areas in the state (Cline and Marshall 1977). Based on the Morgan soil test extraction (Morgan 1941) and soil fertility interpretations for New York (Cornell Cooperative Extension 2006), all sites were classified as high in available $\mathrm{P}$ and high or very high in magnesium $(\mathrm{Mg}$ ). Two sites were low in $\mathrm{K}$ (Columbia and Essex County), two were medium in K (St Lawrence and Cayuga County), and the remaining sites were high in available K. Previous field histories varied from continuous corn to corn in rotation with wheat, barley or grass/legume sods. In St Lawrence County, the trial followed $\mathrm{S} \times \mathrm{S}$ (Table 1). The 2004 growing season (Table 2) was characterized by mean monthly temperatures that were slightly below average and rainfall that was above average for five of the six sites; the Columbia County site was warmer than average and received precipitation well-above average.

\section{Experimental design and crop management}

Each trial was conducted as a complete randomized block design with six treatments and four replicates. Plots were 18 by $3.6 \mathrm{~m}$. The trial in Columbia County received about $52360 \mathrm{~L}$ manure ha ${ }^{-1}$ plowed down within $5 \mathrm{hr}$ just prior to planting. This resulted in an application of $134 \mathrm{~kg} \mathrm{ha}^{-1}$ available $\mathrm{N}$ assuming $65 \%$ availability of inorganic $\mathrm{N}$ and an organic $\mathrm{N}$ release of $35 \%$ (Ketterings et al. 2003). This trial had $5 \mathrm{~N}$ treatments $(0,56,112,168$, and $224 \mathrm{~kg} \mathrm{~N} \mathrm{ha}^{-1}$ per cut) as well as a control that had not received any manure or fertilizer since 2002. Nitrogen applications were carried out using urea in a broadcast application. All other trials had $6 \mathrm{~N}$ fertilizer treatments $(0$, $56,112,168,224$, and $280 \mathrm{~kg} \mathrm{~N} \mathrm{ha}^{-1}$ per cut) and $\mathrm{N}$ applications in the form of ammonium sulfate $(21 \% \mathrm{~N})$ to minimize volatilization losses. The site in Jefferson County was fertilized by the producer with $41 \mathrm{~kg} \mathrm{~N} \mathrm{ha}^{-1}$ prior to establishment of the trial in the spring of 2004.

The Columbia County site received the equivalent of $94 \mathrm{~kg} \mathrm{P}_{2} \mathrm{O}_{5} \mathrm{ha}^{-1}$ with the manure application. Each of the other sites was fertilized with $22-50 \mathrm{~kg} \mathrm{P}_{2} \mathrm{O}_{5} \mathrm{ha}^{-1}$ (Table 1). The two sites that were low in $\mathrm{K}$ received ample $\mathrm{K}$ with fertilizer or manure to overcome $\mathrm{K}$ deficiency. At the other sites 22-67 $\mathrm{kg} \mathrm{K}_{2} \mathrm{O}$ ha $^{-1}$ were applied (Table 1).

The BMR S $\times$ S was planted in June, 2004, using John Deere grain drills and a seeding rate of $67 \mathrm{~kg}$ of seed $\mathrm{ha}^{-1}$. Two harvests were obtained at five of the six sites. Harvest areas were $0.9 \mathrm{~m}$ wide and 7-9 $\mathrm{m}$ long with the exception of the on-farm trials in Columbia and Jefferson County where harvested areas were $0.9 \mathrm{~m}$ wide and $1.5 \mathrm{~m}$ long. The Jefferson country trial had one cut only, due to late planting and limited growth following first cutting.

Harvest was initiated when the plots that had received $168 \mathrm{~kg} \mathrm{~N} \mathrm{ha}^{-1}$ had reached a stand height of $90-110 \mathrm{~cm}$ to optimize forage quality (Kilcer et al. 2005). Stand height was measured from the surface of the soil to the horizontal curve of the tallest emerged leaf. Cutting height was $7.5-9.0 \mathrm{~cm}$. Fresh weights were determined and subsamples were taken to determine moisture content and nutrient concentration. Samples were dried for three days at $65^{\circ} \mathrm{C}$ and ground using a UDY cyclone sample mill (UDY Corporation, Fort Collins, CO, USA) with a $1.0-\mathrm{mm}$ screen.

\section{Forage analyses}

All forage analyses were carried out at the Dairy One Forage Testing Laboratory, Ithaca, NY, USA. Nitrogen was determined by dry combustion (Leco Instruments, Inc., St Joseph, MI, USA). For all other nutrients, samples were dry ashed for $4 \mathrm{~h}$ at $500{ }^{\circ} \mathrm{C}$, cooled, and then dried again on a $100-120^{\circ} \mathrm{C}$ hot plate after addition of $3 \mathrm{ml}$ of $6 \mathrm{~N} \mathrm{HCl}$ (Greweling 1976). Ashed samples were extracted in dilute acid $\left(1.5 \mathrm{NHNO}_{3}\right.$ and $0.5 \mathrm{~N} \mathrm{HCl}$ ), and plant $\mathrm{P}, \mathrm{K}, \mathrm{Ca}$, and $\mathrm{Mg}$ concentrations in the extract were determined using a Thermo Jarrel Ash IRIS Advantage Inductively Coupled Plasma Radial Spectrometer (Jarrell Ash, Franklin, MA, USA).

\section{Soil analyses}

Soil samples $(0-20 \mathrm{~cm})$ were taken at planting, dried at $65{ }^{\circ} \mathrm{C}$, ground and passed through a $2-\mathrm{mm}$ sieve prior to analyses. All soil fertility analyses were performed at the Cornell Nutrient Analysis Laboratory, Ithaca, NY, USA. 


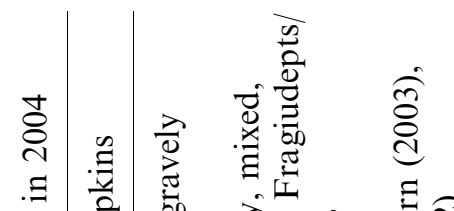

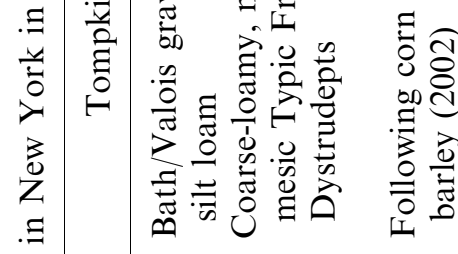

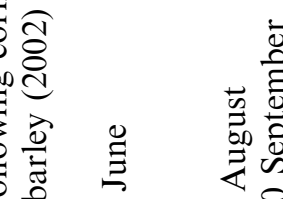

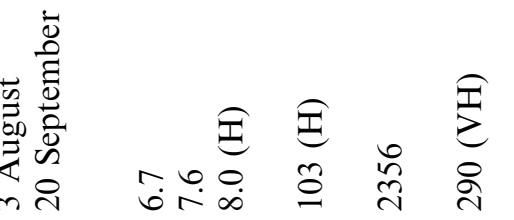

莺

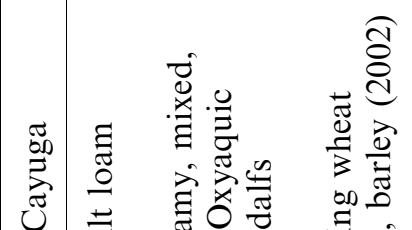

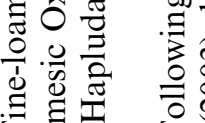

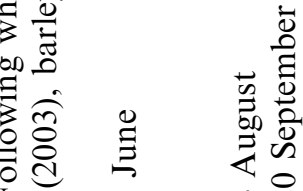

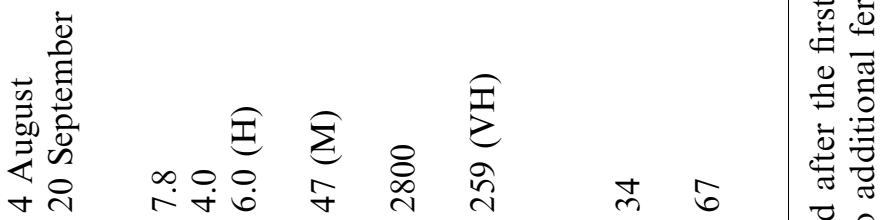

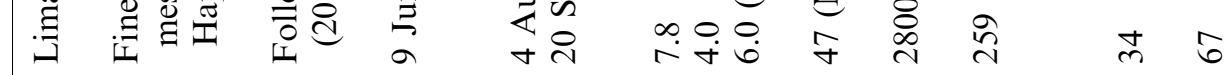

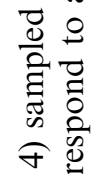

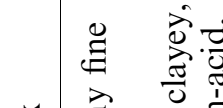

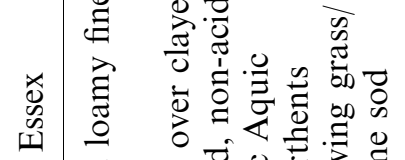

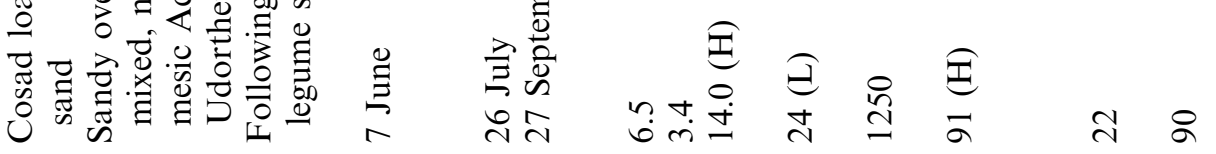

$\|$ 웅



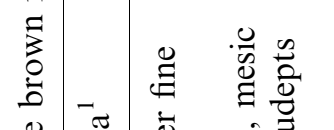

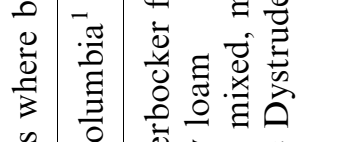

$\stackrel{\infty}{\stackrel{\infty}{*}} \overline{0}$

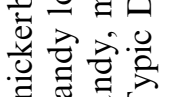



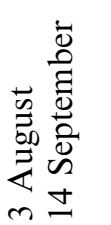

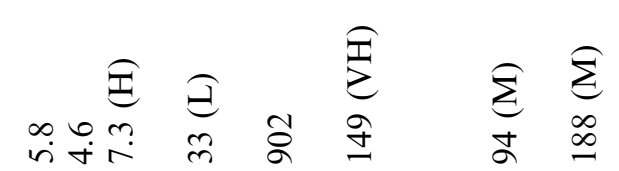

党:

乙至

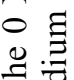

苛

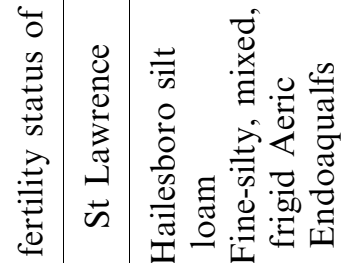

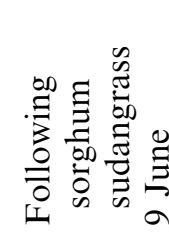

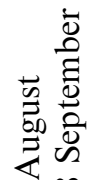

要

:

范

$=0 \stackrel{0}{=}$

年

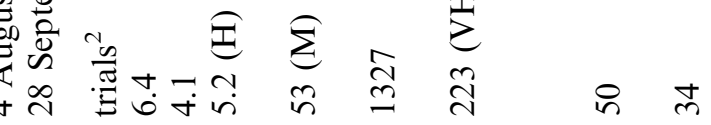
4

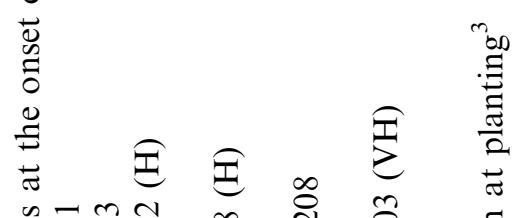

.

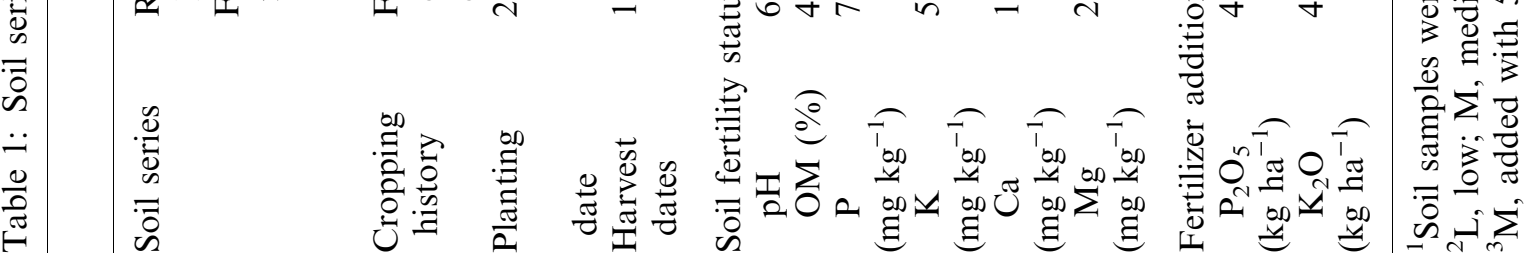

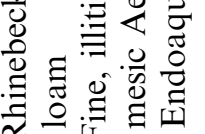

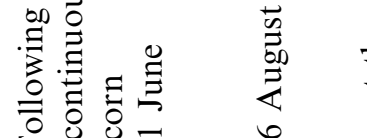

章

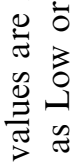

胥苞

牙

$\Xi$ 荡

․ํำ

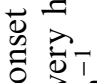

吾 
Table 2: Monthly precipitation and temperature for the six sites where brown midrib sorghum $\times$ sudangrass trials were conducted in New York in 2004

\begin{tabular}{|c|c|c|c|c|}
\hline & June & July & August & September \\
\hline \multicolumn{5}{|c|}{ Total monthly precipitation $(\mathrm{cm})$} \\
\hline Jefferson & 6.6 & 9.3 & 11.0 & 8.5 \\
\hline St Lawrence & 8.7 & 13.5 & 13.8 & $3.0^{1}$ \\
\hline Columbia & 8.7 & 15.4 & 18.6 & 22.0 \\
\hline Essex & 6.3 & 9.8 & $\mathrm{NA}^{2}$ & $\mathrm{NA}^{2}$ \\
\hline Cayuga & 4.4 & 13.9 & 14.1 & 10.5 \\
\hline Tompkins & 6.7 & 17.3 & $\mathrm{NA}^{2}$ & 11.4 \\
\hline \multicolumn{5}{|c|}{ Average monthly temperature $\left({ }^{\circ} \mathrm{C}\right)$} \\
\hline Jefferson & 15.7 & 19.6 & 18.3 & 16.3 \\
\hline St Lawrence & 16.8 & 20.3 & 18.3 & $\mathrm{NA}^{2}$ \\
\hline Columbia & 18.8 & 21.5 & 21.1 & 18.1 \\
\hline Essex & 17.4 & 20.9 & $\mathrm{NA}^{2}$ & 17.6 \\
\hline Cayuga & 18.1 & 20.8 & 20.0 & 18.3 \\
\hline Tompkins & 16.6 & 19.4 & $\mathrm{NA}^{2}$ & 17.4 \\
\hline
\end{tabular}

Data were obtained from the weather station nearest to the sites (Northeast Regional Climate Center 2006).

${ }^{1} 2$ days of recording missing.

${ }^{2}$ Eight or more days of recording missing.

Soil organic matter was determined by loss on ignition (Nelson and Sommers 1996). Soil pH was measured in a $1: 1(\mathrm{w} / \mathrm{v})$ water extract. The Morgan solution consisting of $1 \mathrm{~N}$ sodium acetate buffered at $\mathrm{pH} 4.8$ (Morgan 1941) is the basis for fertility recommendations in New York State, Massachusetts, and Rhode Island. This test is also the basis for fertility recommendations in Ireland (Daly and Casey 2003). A slightly modified procedure that uses ammonium acetate instead of sodium acetate, also buffered at $\mathrm{pH} 4.8$ (McIntosh 1969), is used for fertilizer recommendations in Vermont, Maine and Connecticut. All plots were analysed for Morgan extractable $\mathrm{P}, \mathrm{K}, \mathrm{Ca}$ and $\mathrm{Mg}$ by shaking dried samples in a $1: 5(\mathrm{v} / \mathrm{v})$ ratio for $15 \mathrm{~min}$ and filtering the extract through a Whatman no. 2 equivalent filter paper. The extracts were analysed using a JY70 Type II Inductively Coupled Plasma Atomic Emission Spectrometer (Jobin Yvon, Edison, NJ, USA).

\section{Statistical analyses}

Trials were analysed individually using PROC MIXED of SAS Institute Inc. (1999) with block effects as random effects and $\mathrm{N}$ rate as fixed effect. Mean differences were considered significant if $\mathrm{P} \leq 0.05$. PROC REG was used to determine the relationships between dry matter yield (independent variable) and nutrient removal (dependent variable).

\section{Results and Discussion}

\section{Nutrient concentrations}

The mean $\mathrm{N}$ concentration across all sites and cutting was $19.8 \mathrm{~g} \mathrm{~N} \mathrm{~kg}^{-1}$ with a standard deviation of $6.8 \mathrm{~g} \mathrm{~kg}^{-1}$. Concentrations $<10 \mathrm{~g} \mathrm{~N} \mathrm{~kg}^{-1}$ dry matter $(\mathrm{DM})$ were measured for first cutting when no additional $\mathrm{N}$ was added in Essex County and when $\mathrm{N}$ applications were $112 \mathrm{~kg} \mathrm{ha}^{-1}$ or less in Jefferson County (Table 3). This may be due to higher DM yields for first cutting in those two counties (data not shown). The highest $\mathrm{N}$ concentrations $\left(>30 \mathrm{~g} \mathrm{~kg}^{-1}\right)$ were obtained in the manured trial in Columbia County. These concentrations are substantially higher than the $13 \mathrm{~g} \mathrm{~kg}^{-1}$ reported as average $\mathrm{N}$ concentration for maize silage but lower than the $82 \mathrm{~g} \mathrm{~kg}^{-1}$ reported by the Dairy One Forage Laboratory, Ithaca, NY, for high protein feeds such as soybean meal (Dairy One 2006). Forage $\mathrm{N}$ concentrations increased with $\mathrm{N}$ application (Table 3) but varied from location to location, consistent with observations by Beyaert and Roy (2005), who attributed variability over sites and years to water stress.

Phosphorus concentrations ranged from $<2.0 \mathrm{~g} \mathrm{~kg}^{-1}$ in the Jefferson County site to $4.4 \mathrm{~g} \mathrm{~kg}^{-1}$ in second cutting in the Columbia County site when no fertilizer $\mathrm{N}$ or manure was applied (Table 3), with a mean $\mathrm{P}$ concentration across all sites of $2.9 \mathrm{~g} \mathrm{P} \mathrm{kg}^{-1}$ (standard deviation $=0.7 \mathrm{~g} \mathrm{P} \mathrm{kg}^{-1}$ ). These ranges and the mean concentration are comparable with those reported in Ketterings et al. (2004) and higher than the $2.3 \mathrm{~g} \mathrm{~kg}^{-1}$ average $\mathrm{P}$ concentration reported for maize across 71 sites throughout NY in 2001-2003 (Ketterings et al. 2005b) and $2.4 \mathrm{~g} \mathrm{~kg}^{-1}$ reported as a 5 -year average for maize by the Dairy One (2006). The BMR S $\times$ S P concentrations are similar to those reported for small grains, clovers and other 
Table 3: Nitrogen $(\mathrm{N})$, phosphorus $(\mathrm{P})$, potassium $(\mathrm{K})$, calcium $(\mathrm{Ca})$ and magnesium $(\mathrm{Mg})$ concentrations of first and second cutting as impacted by $\mathrm{N}$ fertilization rate in six BMR sorghum $\times$ sudangrass studies in New York ${ }^{1}($ see Table 1 for a description of the sites)

\begin{tabular}{|c|c|c|c|c|c|c|c|c|c|c|c|c|}
\hline \multirow{2}{*}{$\begin{array}{l}\mathrm{Kg} \mathrm{N}_{\mathrm{ha}^{-1} \mathrm{cut}^{-1}}\end{array}$} & \multicolumn{2}{|c|}{ Jefferson } & \multicolumn{2}{|c|}{ St Lawrence } & \multicolumn{2}{|c|}{ Columbia $^{2}$} & \multicolumn{2}{|c|}{ Essex } & \multicolumn{2}{|c|}{ Cayuga } & \multicolumn{2}{|c|}{ Tompkins } \\
\hline & First & Second & First & Second & First & Second & First & Second & First & Second & First & Second \\
\hline \multicolumn{13}{|c|}{ Nitrogen ( $\mathrm{g} \mathrm{N} \mathrm{kg}^{-1}$ of dry matter) } \\
\hline 0 & - & - & $16.3 \mathrm{c}$ & $13.5 \mathrm{~d}$ & $17.2 \mathrm{~d}$ & $13.1 \mathrm{c}$ & $9.8 \mathrm{~b}$ & $12.6 \mathrm{~b}$ & $15.4 \mathrm{~b}$ & $14.1 \mathrm{c}$ & $15.4 \mathrm{a}$ & $15.9 \mathrm{~d}$ \\
\hline $0+\mathrm{M}$ & - & - & - & - & $22.8 \mathrm{~cd}$ & $18.3 \mathrm{bc}$ & - & - & - & - & - & - \\
\hline 43 & $9.1 \mathrm{bc}$ & - & - & - & - & - & - & - & - & - & - & - \\
\hline 56 & $8.4 \mathrm{c}$ & - & $19.7 \mathrm{bc}$ & $15.3 \mathrm{~cd}$ & $28.9 \mathrm{bc}$ & $22.1 \mathrm{~b}$ & $14.2 \mathrm{ab}$ & $11.6 \mathrm{~b}$ & $16.4 \mathrm{~b}$ & $13.4 \mathrm{c}$ & $14.2 \mathrm{a}$ & $18.3 \mathrm{~cd}$ \\
\hline 112 & $8.9 \mathrm{bc}$ & - & $18.1 \mathrm{bc}$ & $19.0 \mathrm{bc}$ & $34.0 \mathrm{ab}$ & $30.3 \mathrm{a}$ & $20.2 \mathrm{ab}$ & $14.6 \mathrm{ab}$ & $18.7 \mathrm{ab}$ & $14.2 \mathrm{c}$ & $17.8 \mathrm{a}$ & $21.3 \mathrm{bc}$ \\
\hline 168 & $13.9 \mathrm{~b}$ & - & $23.2 \mathrm{ab}$ & $22.9 \mathrm{ab}$ & $32.4 \mathrm{ab}$ & $32.7 \mathrm{a}$ & $20.5 \mathrm{ab}$ & $15.7 \mathrm{a}$ & $19.8 \mathrm{ab}$ & $18.3 \mathrm{~b}$ & $21.5 \mathrm{a}$ & $22.0 \mathrm{bc}$ \\
\hline 224 & $19.9 \mathrm{a}$ & - & $27.0 \mathrm{a}$ & $25.1 \mathrm{a}$ & $36.2 \mathrm{a}$ & $33.2 \mathrm{a}$ & $22.4 \mathrm{a}$ & $16.0 \mathrm{a}$ & $21.9 \mathrm{a}$ & $18.7 \mathrm{~b}$ & $22.2 \mathrm{a}$ & $25.0 \mathrm{ab}$ \\
\hline 280 & $22.3 \mathrm{a}$ & - & $27.2 \mathrm{a}$ & $26.4 \mathrm{a}$ & - & - & $19.2 \mathrm{ab}$ & $17.4 \mathrm{a}$ & $23.2 \mathrm{a}$ & $22.6 \mathrm{a}$ & $17.2 \mathrm{a}$ & $28.1 \mathrm{a}$ \\
\hline \multicolumn{13}{|c|}{ Phosphorus (g P kg-1 of dry matter) } \\
\hline 0 & - & - & $3.2 \mathrm{a}$ & $4.3 \mathrm{a}$ & $3.0 \mathrm{ab}$ & $4.4 \mathrm{a}$ & $2.6 \mathrm{a}$ & $4.1 \mathrm{a}$ & $3.0 \mathrm{a}$ & $3.5 \mathrm{a}$ & $4.0 \mathrm{a}$ & $3.8 \mathrm{a}$ \\
\hline $0+\mathrm{M}$ & - & - & - & - & $3.3 \mathrm{a}$ & $4.0 \mathrm{ab}$ & - & - & - & - & - & - \\
\hline 43 & $1.9 \mathrm{a}$ & - & - & - & - & - & - & - & - & - & - & - \\
\hline 56 & $1.6 \mathrm{ab}$ & - & $3.2 \mathrm{a}$ & $3.7 \mathrm{~b}$ & $3.0 \mathrm{ab}$ & $3.8 \mathrm{~b}$ & $2.7 \mathrm{a}$ & $3.0 \mathrm{~b}$ & $2.7 \mathrm{a}$ & $2.9 \mathrm{~b}$ & $3.6 \mathrm{a}$ & $3.6 \mathrm{ab}$ \\
\hline 112 & $1.2 \mathrm{~b}$ & - & $2.9 \mathrm{a}$ & $3.2 \mathrm{c}$ & $2.7 \mathrm{ab}$ & $3.5 \mathrm{~b}$ & $2.2 \mathrm{a}$ & $2.4 \mathrm{~b}$ & $2.9 \mathrm{a}$ & $2.5 \mathrm{bc}$ & $3.3 \mathrm{a}$ & $3.5 \mathrm{ab}$ \\
\hline 168 & $1.4 \mathrm{~b}$ & - & $3.1 \mathrm{a}$ & $3.0 \mathrm{c}$ & $2.5 \mathrm{~b}$ & $3.5 \mathrm{~b}$ & $2.2 \mathrm{a}$ & $2.3 \mathrm{~b}$ & $2.8 \mathrm{a}$ & $2.4 \mathrm{bc}$ & $3.4 \mathrm{a}$ & $3.2 \mathrm{~b}$ \\
\hline 224 & $1.4 \mathrm{~b}$ & - & $2.9 \mathrm{a}$ & $2.9 \mathrm{c}$ & $2.8 \mathrm{ab}$ & $3.7 \mathrm{~b}$ & $2.3 \mathrm{a}$ & $2.0 \mathrm{~b}$ & $2.7 \mathrm{a}$ & $2.4 \mathrm{c}$ & $3.4 \mathrm{a}$ & $3.2 \mathrm{~b}$ \\
\hline 280 & $1.4 \mathrm{~b}$ & - & $2.9 \mathrm{a}$ & $2.9 \mathrm{c}$ & - & - & $2.3 \mathrm{a}$ & $2.4 \mathrm{~b}$ & $2.6 \mathrm{a}$ & $2.4 \mathrm{c}$ & $3.6 \mathrm{a}$ & $3.1 \mathrm{~b}$ \\
\hline \multicolumn{13}{|c|}{ Potassium ( $\mathrm{g} \mathrm{K} \mathrm{kg}^{-1}$ of dry matter) } \\
\hline 0 & - & - & $27.3 \mathrm{a}$ & $17.6 \mathrm{a}$ & $23.9 \mathrm{a}$ & $18.7 \mathrm{ab}$ & $21.2 \mathrm{a}$ & $16.9 \mathrm{a}$ & $28.3 \mathrm{a}$ & $19.9 \mathrm{a}$ & $29.2 \mathrm{a}$ & $22.7 \mathrm{a}$ \\
\hline $0+\mathrm{M}$ & - & - & - & - & $29.3 \mathrm{a}$ & $20.2 \mathrm{a}$ & - & - & - & - & - & - \\
\hline 43 & $23.3 \mathrm{a}$ & - & - & - & - & - & - & - & - & - & - & - \\
\hline 56 & $22.1 \mathrm{a}$ & - & $25.0 \mathrm{a}$ & $16.6 \mathrm{a}$ & $30.1 \mathrm{a}$ & $17.0 \mathrm{ab}$ & $17.0 \mathrm{ab}$ & $9.7 \mathrm{~b}$ & $25.9 \mathrm{a}$ & $18.2 \mathrm{ab}$ & $28.7 \mathrm{a}$ & $22.9 \mathrm{a}$ \\
\hline 112 & $20.7 \mathrm{a}$ & - & $25.5 \mathrm{a}$ & $15.6 \mathrm{a}$ & $28.3 \mathrm{a}$ & $13.8 \mathrm{~b}$ & $15.9 \mathrm{ab}$ & $9.2 \mathrm{~b}$ & $27.5 \mathrm{a}$ & $16.2 \mathrm{bc}$ & $28.2 \mathrm{a}$ & $22.7 \mathrm{a}$ \\
\hline 168 & $19.8 \mathrm{a}$ & - & $23.8 \mathrm{a}$ & $15.4 \mathrm{a}$ & $22.9 \mathrm{a}$ & $16.4 \mathrm{ab}$ & $14.2 \mathrm{ab}$ & $9.3 \mathrm{~b}$ & $26.6 \mathrm{a}$ & $15.4 \mathrm{c}$ & $29.0 \mathrm{a}$ & $19.6 \mathrm{a}$ \\
\hline 224 & $18.0 \mathrm{a}$ & - & $23.0 \mathrm{a}$ & $16.3 \mathrm{a}$ & $30.1 \mathrm{a}$ & $16.7 \mathrm{ab}$ & $15.8 \mathrm{ab}$ & $9.1 \mathrm{~b}$ & $25.0 \mathrm{a}$ & $15.3 \mathrm{c}$ & $27.7 \mathrm{a}$ & $20.8 \mathrm{a}$ \\
\hline 280 & $21.9 \mathrm{a}$ & - & $23.7 \mathrm{a}$ & $16.2 \mathrm{a}$ & - & - & $11.4 \mathrm{~b}$ & $8.1 \mathrm{~b}$ & $25.0 \mathrm{a}$ & $15.7 \mathrm{c}$ & $28.7 \mathrm{a}$ & $23.9 \mathrm{a}$ \\
\hline \multicolumn{13}{|c|}{ Calcium ( $\mathrm{g} \mathrm{Ca} \mathrm{kg}^{-1}$ of dry matter) } \\
\hline 0 & - & - & $5.3 \mathrm{~b}$ & $5.7 \mathrm{a}$ & $5.2 \mathrm{a}$ & $5.2 \mathrm{~b}$ & $6.2 \mathrm{~b}$ & $6.2 \mathrm{a}$ & $5.3 \mathrm{c}$ & $4.9 \mathrm{~b}$ & $4.0 \mathrm{~b}$ & $3.4 \mathrm{a}$ \\
\hline $0+\mathrm{M}$ & - & - & - & - & $5.3 \mathrm{a}$ & $5.3 \mathrm{~b}$ & - & - & - & - & - & - \\
\hline 43 & $4.1 \mathrm{a}$ & - & - & - & - & - & - & - & - & - & - & - \\
\hline 56 & $4.1 \mathrm{a}$ & - & $5.6 \mathrm{~b}$ & $6.3 \mathrm{a}$ & $5.8 \mathrm{a}$ & $5.7 \mathrm{ab}$ & $7.6 \mathrm{ab}$ & $6.6 \mathrm{a}$ & $5.6 \mathrm{bc}$ & 5.3 & $4.0 \mathrm{ab}$ & $3.5 \mathrm{a}$ \\
\hline 112 & $3.9 \mathrm{a}$ & - & $5.4 \mathrm{~b}$ & $6.1 \mathrm{a}$ & $6.0 \mathrm{a}$ & $6.7 \mathrm{a}$ & $8.1 \mathrm{a}$ & $7.1 \mathrm{a}$ & $5.8 \mathrm{abc}$ & $5.6 \mathrm{abc}$ & $4.2 \mathrm{ab}$ & $3.7 \mathrm{a}$ \\
\hline 168 & $4.0 \mathrm{a}$ & - & $5.5 \mathrm{~b}$ & $6.1 \mathrm{a}$ & $5.1 \mathrm{a}$ & $6.8 \mathrm{a}$ & $8.3 \mathrm{a}$ & $7.0 \mathrm{a}$ & $5.9 \mathrm{ab}$ & $5.8 \mathrm{ab}$ & $4.2 \mathrm{ab}$ & $3.5 \mathrm{a}$ \\
\hline 224 & $4.1 \mathrm{a}$ & - & $6.0 \mathrm{ab}$ & $6.0 \mathrm{a}$ & $6.4 \mathrm{a}$ & $6.6 \mathrm{ab}$ & $8.3 \mathrm{a}$ & $6.9 \mathrm{a}$ & $6.1 \mathrm{ab}$ & $5.9 \mathrm{ab}$ & $4.8 \mathrm{a}$ & $3.6 \mathrm{a}$ \\
\hline 280 & $4.5 \mathrm{a}$ & - & $6.4 \mathrm{a}$ & $6.3 \mathrm{a}$ & - & - & $8.6 \mathrm{a}$ & $7.5 \mathrm{a}$ & $6.2 \mathrm{a}$ & $6.3 \mathrm{a}$ & $4.0 \mathrm{ab}$ & $3.8 \mathrm{a}$ \\
\hline \multicolumn{13}{|c|}{ Magnesium ( $\mathrm{g} \mathrm{Mg} \mathrm{kg}^{-1}$ of dry matter) } \\
\hline 0 & - & - & $3.6 \mathrm{c}$ & $3.8 \mathrm{~b}$ & $5.2 \mathrm{~b}$ & $5.9 \mathrm{~b}$ & $3.2 \mathrm{a}$ & $3.6 \mathrm{a}$ & $3.1 \mathrm{c}$ & $2.3 \mathrm{~d}$ & $2.1 \mathrm{a}$ & $2.3 \mathrm{~d}$ \\
\hline $0+\mathrm{M}$ & - & - & - & - & $5.8 \mathrm{~b}$ & $6.5 \mathrm{~b}$ & - & - & - & - & - & - \\
\hline 43 & $2.0 \mathrm{a}$ & - & - & - & - & - & - & - & - & - & - & - \\
\hline 56 & $2.0 \mathrm{a}$ & - & $3.9 \mathrm{c}$ & $4.7 \mathrm{a}$ & $6.6 \mathrm{ab}$ & $7.5 \mathrm{ab}$ & $4.5 \mathrm{a}$ & $4.7 \mathrm{a}$ & $3.6 \mathrm{bc}$ & $3.0 \mathrm{c}$ & $2.1 \mathrm{a}$ & $2.6 \mathrm{~cd}$ \\
\hline 112 & $2.0 \mathrm{a}$ & - & $4.2 \mathrm{bc}$ & $5.1 \mathrm{a}$ & $6.4 \mathrm{ab}$ & $9.1 \mathrm{a}$ & $4.2 \mathrm{a}$ & $4.1 \mathrm{a}$ & $3.8 \mathrm{ab}$ & $3.3 \mathrm{bc}$ & $2.4 \mathrm{a}$ & $2.9 \mathrm{bc}$ \\
\hline 168 & $2.2 \mathrm{a}$ & - & $4.7 \mathrm{ab}$ & $5.0 \mathrm{a}$ & $5.9 \mathrm{ab}$ & $9.5 \mathrm{a}$ & $4.4 \mathrm{a}$ & $3.8 \mathrm{a}$ & $3.9 \mathrm{ab}$ & $3.8 \mathrm{ab}$ & $2.8 \mathrm{a}$ & $3.1 \mathrm{abc}$ \\
\hline 224 & $2.0 \mathrm{a}$ & - & $4.8 \mathrm{ab}$ & $4.7 \mathrm{a}$ & $7.9 \mathrm{a}$ & $9.5 \mathrm{a}$ & $4.3 \mathrm{a}$ & $3.7 \mathrm{a}$ & $4.0 \mathrm{ab}$ & $3.6 \mathrm{abc}$ & $3.0 \mathrm{a}$ & $3.3 \mathrm{ab}$ \\
\hline 280 & $2.0 \mathrm{a}$ & - & $5.0 \mathrm{a}$ & $4.8 \mathrm{a}$ & - & - & $4.6 \mathrm{a}$ & $4.3 \mathrm{a}$ & $4.2 \mathrm{a}$ & $3.9 \mathrm{a}$ & $2.4 \mathrm{a}$ & $3.5 \mathrm{a}$ \\
\hline
\end{tabular}

${ }^{1}$ Average values within columns with different letters $(a, b, c)$ are statistically different $(\mathrm{P} \leq 0.05)$.

${ }^{2}$ This site received manure at a rate of $52360 \mathrm{~L} \mathrm{ha}^{-1}$ plowed down within $5 \mathrm{hr}$ resulting in an application of $134 \mathrm{~kg}$ available $\mathrm{N} \mathrm{ha}^{-1}, 94 \mathrm{~kg} \mathrm{P}_{2} \mathrm{O}_{5}$ and $188 \mathrm{~kg} \mathrm{~K}_{2} \mathrm{O} \mathrm{ha}{ }^{-1}$.

legumes by Brink et al. (2001). Nitrogen application significantly reduced $\mathrm{P}$ concentrations in either the first or the second cutting or in both, depending on location. This is also consistent with findings reported for BMR $\mathrm{S} \times \mathrm{S}$ trials conducted in NY in 20022003 (Ketterings et al. 2004). 
Mean $\mathrm{K}$ concentrations decreased with $\mathrm{N}$ application, although differences were not statistically significant in the Jefferson, St Lawrence and Tompkins County sites. Potassium concentrations were $<25 \mathrm{~g} \mathrm{~kg}^{-1}$ for second cuttings at all sites, making second cuttings suitable for non-lactating cows, even at the site where manure was applied (Table 3). In previous studies with BMR $\mathrm{S} \times \mathrm{S}$ (Ketterings et al. 2005a), we concluded that $\mathrm{K}$ availability and concentration had little influence on yield, while $\mathrm{N}$ fertilizer greatly increased yield. Cherney et al. (1998) summarized literature on elemental concentration shifts in grass herbage when either $\mathrm{N}$ or $\mathrm{K}$ was applied and concluded that $\mathrm{N}$ fertilizer will increases both $\mathrm{K}$ uptake and plant $\mathrm{K}$ concentration if there is enough $\mathrm{K}$ available in the soil. When $\mathrm{K}$ becomes limiting, increasing $\mathrm{N}$ fertilizer results in a decreased plant $\mathrm{K}$ concentration (a dilution effect). Our results are consistent with such a decrease in $\mathrm{K}$ availability.

Manure was only applied at the onset of the trial in Columbia County, and forage $\mathrm{K}$ concentrations will likely exceed recommended levels for nonlactating cows if a second manure application takes place following first cutting. The $\mathrm{K}$ concentrations in the second cutting in Essex County may suggest a $\mathrm{K}$ deficiency, although $\mathrm{K}$ response trials need to be conducted to confirm a deficiency as sufficiency ranges for this crop are not known.

Magnesium concentrations ranged from $2.0 \mathrm{~g} \mathrm{~kg}^{-1}$ in the single cut in Jefferson County site to $9.5 \mathrm{~g} \mathrm{~kg}^{-1}$ in second cutting of the manured site in Columbia County, with a mean across all sites of $4.2 \mathrm{~g} \mathrm{Mg} \mathrm{kg}^{-1}$ (standard deviation = $1.9 \mathrm{~g} \mathrm{~kg}^{-1}$ ). Magnesium concentrations in the forage at the Tompkins County site were comparable with values reported in Ketterings et al. (2005a) for a $\mathrm{N} \times \mathrm{K}$ trial conducted in 2002-2003 in the same county. The mean $\mathrm{Ca}$ concentration across all sites was $5.6 \mathrm{~g} \mathrm{~kg}^{-1}$ (standard deviation = $1.4 \mathrm{~g} \mathrm{~kg}^{-1}$ ). Mean $\mathrm{Ca}$ and $\mathrm{Mg}$ concentrations increased with $\mathrm{N}$ application at all sites except for the Jefferson County site and the Essex County site, where similar trends were observed but means were not statistically significant. An increase in $\mathrm{Ca}$ and $\mathrm{Mg}$ may be due to the decreased $\mathrm{K}$ concentrations with $\mathrm{N}$ addition on these high $\mathrm{Mg}$ fertility soils. These results are consistent with observations by Cherney et al. (1998) for grass forage; N fertilizer tends to increase plant $\mathrm{Mg}$, but has inconsistent effects on plant $\mathrm{Ca}$ concentrations, and $\mathrm{N}$ effects on $\mathrm{Ca}$ and $\mathrm{Mg}$ can be enhanced by limited $\mathrm{K}$ availability.

\section{Nutrient removal}

Nitrogen removal ranged from $30 \mathrm{~kg} \mathrm{~N} \mathrm{ha}^{-1}$ at the lowest $\mathrm{N}$ rate in the one-cut system in Jefferson County to $510 \mathrm{~kg} \mathrm{~N} \mathrm{ha}^{-1}$ with the highest $\mathrm{N}$ additions in the manured site (Table 4). The manured site showed the highest $\mathrm{N}$ uptake increase per Mg DM (43 $\left.\mathrm{kg} \mathrm{N} \mathrm{Mg}^{-1} \mathrm{DM}\right)$, consistent with the $251-393 \mathrm{~kg} \mathrm{~N} \mathrm{ha}^{-1}$ uptake reported for forage $\mathrm{S} \times \mathrm{S}$ by McLaughlin et al. (2004). Dry matter yield accounted for $80-91 \%$ of the variability in $\mathrm{N}$ removal for all sites except the one-cut system in Jefferson County $(47 \%)$ and the site that followed plowdown of a grass/legume sod in Essex County (Table 5). At this site, the low $\mathrm{K}$ fertility status and absence of a second $\mathrm{K}$ application following first cutting, may have caused a $\mathrm{K}$ deficiency that impacted the $\mathrm{N}$ response.

Dry matter yield accounted for $>85 \%$ of the variability in P removal at the sites in St Lawrence, Columbia, Cayuga and Tompkins counties, with $\mathrm{P}$ uptake increases in these four counties ranging from $5.30 \mathrm{~kg} \mathrm{P}_{2} \mathrm{O}_{5} \mathrm{Mg}^{-1} \mathrm{DM}$ in Cayuga County to $7.14 \mathrm{~kg} \mathrm{P}_{2} \mathrm{O}_{5} \mathrm{Mg}^{-1}$ in Tompkins County (Table 5). In the single cut system in Jefferson County, $61 \%$ of the variability in $\mathrm{P}$ removal was explained by dry matter yield while for Essex County, DM production only explained $43 \%$ of the variability, possibly due to a $\mathrm{K}$ deficiency. Phosphorus removal rates in the manured site were only slightly lower than the 101-129 $\mathrm{kg} \mathrm{P}_{2} \mathrm{O}_{5}$ ha $^{-1}$ obtained with forage $\mathrm{S} \times \mathrm{S}$ in swine manure fertilized fields in Mississippi and higher than the other warm season grasses studied by McLaughlin et al. (2004).

Potassium removal rates were substantial, ranging from $98 \mathrm{~kg} \mathrm{~K}_{2} \mathrm{O}$ ha ${ }^{-1}$ without $\mathrm{N}$ addition in the Jefferson County site to almost $500 \mathrm{~kg} \mathrm{ha}^{-1}$ with the highest $\mathrm{N}$ application in the manured site (Table 4). These results suggest that high yields will quickly deplete available soil $\mathrm{K}$.

The $\mathrm{K}$ removal rates at the highest $\mathrm{N}$ treatments in the manure site (Table 5) were similar to those reported for swine manure amended fields in Mississippi (McLaughlin et al. 2004). Dry matter yields explained $80-95 \%$ of the variability in $\mathrm{K}$ removal in four of the six sites. Exceptions were the one-cut system in Jefferson County, where yield explained only $64 \%$ of the variability, and the Essex County site, where the relationship between dry matter yield and $\mathrm{K}$ removal was not significant (Table 5). The latter supports the observations that this site was $\mathrm{K}$ deficient during the second part of the growing season. 
Table 4: Nitrogen $(\mathrm{N})$, phosphorus $(\mathrm{P})$, potassium $(\mathrm{K})$, calcium $(\mathrm{Ca})$ and magnesium $(\mathrm{Mg})$ removal with harvest as impacted by $\mathrm{N}$ fertilization rate in six BMR sorghum $\times$ sudangrass forage studies in New York ${ }^{1}$ (see Table 1 for a description of the sites)

\begin{tabular}{|c|c|c|c|c|c|c|}
\hline $\begin{array}{l}\mathrm{Kg} \mathrm{N} \\
\mathrm{ha}^{-1} \mathrm{cut}^{-1}\end{array}$ & Jefferson & St Lawrence & Columbia $^{2}$ & Essex & Cayuga & Tompkins \\
\hline \multicolumn{7}{|c|}{ Nitrogen $\left(\mathrm{kg} \mathrm{N} \mathrm{ha}^{-1}\right)$} \\
\hline 0 & - & $56 \mathrm{c}$ & $77 \mathrm{~d}$ & $71 \mathrm{~b}$ & $58 \mathrm{~d}$ & $40 \mathrm{~b}$ \\
\hline $0+\mathrm{M}^{2}$ & - & - & $211 \mathrm{c}$ & - & - & - \\
\hline 43 & $30 \mathrm{c}$ & - & - & & & \\
\hline 56 & $34 \mathrm{bc}$ & $102 \mathrm{~b}$ & $320 \mathrm{bc}$ & $140 \mathrm{ab}$ & $99 \mathrm{~cd}$ & $95 \mathrm{~b}$ \\
\hline 112 & $44 \mathrm{bc}$ & $131 \mathrm{~b}$ & $358 \mathrm{~b}$ & $186 \mathrm{a}$ & $125 \mathrm{bc}$ & $166 \mathrm{a}$ \\
\hline 168 & $78 \mathrm{ab}$ & 189 a & $386 \mathrm{~b}$ & $194 \mathrm{a}$ & $153 \mathrm{ab}$ & $179 \mathrm{a}$ \\
\hline 224 & $118 \mathrm{a}$ & $198 \mathrm{a}$ & $510 \mathrm{a}$ & $205 \mathrm{a}$ & $169 \mathrm{a}$ & $228 \mathrm{a}$ \\
\hline 280 & $106 \mathrm{a}$ & $225 \mathrm{a}$ & - & $185 \mathrm{a}$ & $167 \mathrm{a}$ & $222 \mathrm{a}$ \\
\hline \multicolumn{7}{|c|}{ Phosphorus (kg $\left.\mathrm{P}_{2} \mathrm{O}_{5} \mathrm{ha}^{-1}\right)$} \\
\hline 0 & - & $30.0 \mathrm{c}$ & $38.6 \mathrm{~b}$ & $46.4 \mathrm{a}$ & $27.4 \mathrm{~b}$ & $24.1 \mathrm{c}$ \\
\hline $0+\mathrm{M}$ & - & - & $79.1 \mathrm{a}$ & - & - & - \\
\hline 43 & $14.9 \mathrm{a}$ & - & - & - & - & - \\
\hline 56 & $14.7 \mathrm{a}$ & $45.9 \mathrm{~b}$ & $91.2 \mathrm{a}$ & $71.7 \mathrm{a}$ & $41.2 \mathrm{ab}$ & $48.6 \mathrm{~b}$ \\
\hline 112 & $13.8 \mathrm{a}$ & $49.5 \mathrm{ab}$ & $76.7 \mathrm{a}$ & $57.1 \mathrm{a}$ & $47.0 \mathrm{a}$ & $66.6 \mathrm{a}$ \\
\hline 168 & $17.2 \mathrm{a}$ & $57.3 \mathrm{a}$ & $78.1 \mathrm{a}$ & $53.6 \mathrm{a}$ & $47.8 \mathrm{a}$ & $64.3 \mathrm{ab}$ \\
\hline 224 & $19.0 \mathrm{a}$ & $51.5 \mathrm{ab}$ & $101.5 \mathrm{a}$ & $53.2 \mathrm{a}$ & $49.1 \mathrm{a}$ & $72.2 \mathrm{a}$ \\
\hline 280 & $15.9 \mathrm{a}$ & $55.3 \mathrm{ab}$ & - & $55.2 \mathrm{a}$ & $42.4 \mathrm{a}$ & $80.5 \mathrm{a}$ \\
\hline \multicolumn{7}{|c|}{ Potassium $\left(\mathrm{kg} \mathrm{K}_{2} \mathrm{O} \mathrm{ha}^{-1}\right)$} \\
\hline 0 & - & $112 \mathrm{c}$ & $141 \mathrm{c}$ & $171 \mathrm{a}$ & $134 \mathrm{~b}$ & $91 \mathrm{~d}$ \\
\hline $0+\mathrm{M}$ & - & - & $334 \mathrm{ab}$ & - & - & - \\
\hline 43 & $98 \mathrm{a}$ & - & - & - & - & - \\
\hline 56 & $119 \mathrm{a}$ & $155 \mathrm{bc}$ & $402 \mathrm{ab}$ & $190 \mathrm{a}$ & $194 \mathrm{a}$ & $199 \mathrm{c}$ \\
\hline 112 & $131 \mathrm{a}$ & $186 \mathrm{ab}$ & $326 \mathrm{~b}$ & $179 \mathrm{a}$ & $219 \mathrm{a}$ & $283 \mathrm{~b}$ \\
\hline 168 & $143 \mathrm{a}$ & $207 \mathrm{a}$ & $321 \mathrm{~b}$ & $163 \mathrm{a}$ & $219 \mathrm{a}$ & $265 \mathrm{bc}$ \\
\hline 224 & $137 \mathrm{a}$ & $195 \mathrm{ab}$ & $496 \mathrm{a}$ & $169 \mathrm{a}$ & $220 \mathrm{a}$ & $301 \mathrm{ab}$ \\
\hline 280 & $140 \mathrm{a}$ & $215 \mathrm{a}$ & - & $128 \mathrm{a}$ & $200 \mathrm{a}$ & $356 \mathrm{a}$ \\
\hline \multicolumn{7}{|c|}{ Calcium (kg Ca ha $\left.{ }^{-1}\right)$} \\
\hline 0 & - & $19.8 \mathrm{~d}$ & $25.4 \mathrm{c}$ & $41.0 \mathrm{~b}$ & $19.8 \mathrm{c}$ & $9.7 \mathrm{c}$ \\
\hline $0+\mathrm{M}$ & - & - & $52.8 \mathrm{~b}$ & - & - & - \\
\hline 43 & $14.3 \mathrm{a}$ & - & - & - & - & - \\
\hline 56 & $17.1 \mathrm{a}$ & $35.1 \mathrm{c}$ & $69.4 \mathrm{ab}$ & $76.5 \mathrm{a}$ & $35.6 \mathrm{~b}$ & $22.0 \mathrm{~b}$ \\
\hline 112 & $19.0 \mathrm{a}$ & $40.5 \mathrm{bc}$ & $69.0 \mathrm{ab}$ & $79.7 \mathrm{a}$ & $43.1 \mathrm{ab}$ & $33.3 \mathrm{ab}$ \\
\hline 168 & $22.1 \mathrm{a}$ & $48.0 \mathrm{ab}$ & $68.4 \mathrm{ab}$ & $81.0 \mathrm{a}$ & $47.5 \mathrm{a}$ & $32.4 \mathrm{ab}$ \\
\hline 224 & $24.1 \mathrm{a}$ & $45.6 \mathrm{ab}$ & $92.7 \mathrm{a}$ & $81.8 \mathrm{a}$ & $49.6 \mathrm{a}$ & $41.2 \mathrm{a}$ \\
\hline 280 & $21.7 \mathrm{a}$ & $52.6 \mathrm{a}$ & - & $81.5 \mathrm{a}$ & $45.6 \mathrm{ab}$ & $40.8 \mathrm{a}$ \\
\hline \multicolumn{7}{|c|}{ Magnesium $\left(\mathrm{kg} \mathrm{Mg} \mathrm{ha}{ }^{-1}\right)$} \\
\hline 0 & - & $13.2 \mathrm{c}$ & $26.5 \mathrm{c}$ & $22.4 \mathrm{a}$ & $11.2 \mathrm{c}$ & $5.7 \mathrm{~d}$ \\
\hline $0+\mathrm{M}$ & - & - & $60.0 \mathrm{~b}$ & - & - & - \\
\hline 43 & $7.3 \mathrm{a}$ & - & - & - & - & - \\
\hline 56 & $8.3 \mathrm{a}$ & $25.6 \mathrm{~b}$ & $83.4 \mathrm{~b}$ & $50.1 \mathrm{a}$ & $21.7 \mathrm{~b}$ & $13.4 \mathrm{c}$ \\
\hline 112 & $9.9 \mathrm{a}$ & $33.3 \mathrm{ab}$ & $81.9 \mathrm{~b}$ & $44.5 \mathrm{a}$ & $26.7 \mathrm{ab}$ & $22.5 \mathrm{~b}$ \\
\hline 168 & $11.9 \mathrm{a}$ & $40.0 \mathrm{a}$ & $85.8 \mathrm{~b}$ & $43.9 \mathrm{a}$ & $30.9 \mathrm{a}$ & $24.6 \mathrm{ab}$ \\
\hline 224 & $11.4 \mathrm{a}$ & $36.4 \mathrm{a}$ & $120.8 \mathrm{a}$ & $43.2 \mathrm{a}$ & $31.4 \mathrm{a}$ & $30.4 \mathrm{a}$ \\
\hline 280 & $9.7 \mathrm{a}$ & $41.1 \mathrm{a}$ & - & $45.7 \mathrm{a}$ & $29.7 \mathrm{a}$ & $29.0 \mathrm{ab}$ \\
\hline
\end{tabular}

${ }_{1}^{1}$ Average values within columns with different letters $(a, b, c)$ are statistically different $(P \leq 0.05)$.

${ }^{2}$ This site received manure at a rate of $52360 \mathrm{~L} \mathrm{ha}^{-1}$ plowed down within 5 hours resulting in an application of approximately $134 \mathrm{~kg} \mathrm{~N} \mathrm{ha}^{-1}, 94 \mathrm{~kg} \mathrm{P}_{2} \mathrm{O}_{5}$ and $188 \mathrm{~kg} \mathrm{~K}_{2} \mathrm{O} \mathrm{ha}^{-1}$.

Calcium removal increased upon the addition of $\mathrm{N}$, although increases were not significant in the Jefferson County trial. Calcium removal increased from $3.86 \mathrm{~kg} \mathrm{Mg}^{-1}$ in Jefferson County to $9.45 \mathrm{~kg} \mathrm{Mg}^{-1} \mathrm{DM}$ in Essex County, with dry matter yield explaining $81-97 \%$ of the variability 
Table 5: Linear regression (nutrient removal $=$ intercept + slope $\times$ yield) for phosphorus, potassium, calcium and magnesium for six New York brown midrib sorghum $\times$ sudangrass $\mathrm{N}$ rate trials

\begin{tabular}{|c|c|c|c|c|}
\hline & Intercept $(\mathrm{P}>\mathrm{F})$ & Slope $(\mathrm{P}>\mathrm{F})$ & $\begin{array}{c}\text { Model } \\
\text { adjusted R }\end{array}$ & $\begin{array}{l}\text { Model } \\
\mathrm{P}>\mathrm{F}\end{array}$ \\
\hline \multicolumn{5}{|c|}{ Nitrogen $\left(\mathrm{kg} \mathrm{N}^{-1}\right)$ removal with harvest $\left(\mathrm{Mg} \mathrm{ha}^{-1}\right.$, first and second cut combined $)$} \\
\hline Jefferson & $-37.50(0.1250)$ & $22.03(0.0001)$ & 0.47 & $<0.0001$ \\
\hline St Lawrence & $-73.90(0.0010)$ & $32.87(<0.0001)$ & 0.86 & $<0.0001$ \\
\hline Columbia & $-137.47(0.0003)$ & $42.05(<0.0001)$ & 0.90 & $<0.0001$ \\
\hline Essex & $-57.82(0.2265)$ & $22.42(<0.0001)$ & 0.49 & $<0.0001$ \\
\hline Cayuga & $-31.57(0.0766)$ & $23.07(<0.0001)$ & 0.80 & $<0.0001$ \\
\hline Tompkins & $-34.38(0.0338)$ & $24.99(<0.0001)$ & 0.91 & $<0.0001$ \\
\hline \multicolumn{5}{|c|}{ Phosphorus $\left(\mathrm{kg} \mathrm{P}_{2} \mathrm{O}_{5} \mathrm{ha}^{-1}\right)$ removal with harvest $\left(\mathrm{Mg} \mathrm{ha}^{-1}\right.$, first and second cut combined) } \\
\hline Jefferson & $3.19(0.1574)$ & $2.64(<0.0001)$ & 0.61 & $<0.0001$ \\
\hline St Lawrence & $11.34(<0.0001)$ & $5.42(<0.0001)$ & 0.93 & $<0.0001$ \\
\hline Columbia & $5.60(0.3831)$ & $6.76(<0.0001)$ & 0.86 & $<0.0001$ \\
\hline Essex & $5.77(0.6346)$ & $5.11(0.0003)$ & 0.43 & 0.0003 \\
\hline Cayuga & $5.63(0.0483)$ & $5.30(<0.0001)$ & 0.90 & $<0.0001$ \\
\hline Tompkins & $5.47(0.0695)$ & $7.14(<0.0001)$ & 0.96 & $<0.0001$ \\
\hline \multicolumn{5}{|c|}{ Potassium ( $\left.\mathrm{kg} \mathrm{K}_{2} \mathrm{O} \mathrm{ha}^{-1}\right)$ removal with harvest $\left(\mathrm{Mg} \mathrm{ha}^{-1}\right.$, first and second cut combined) } \\
\hline Jefferson & $25.46(0.1337)$ & $21.31(<0.0001)$ & 0.64 & $<0.0001$ \\
\hline St Lawrence & $27.12(0.0043)$ & $22.17(<0.0001)$ & 0.94 & $<0.0001$ \\
\hline Columbia & $-66.76(0.1467)$ & $37.88(<0.0001)$ & 0.80 & $<0.0001$ \\
\hline Essex & - & - & - & 0.2588 \\
\hline Cayuga & $49.28(0.0003)$ & $21.34(<0.001)$ & 0.89 & $<0.0001$ \\
\hline Tompkins & $6.38(0.6464)$ & $32.18(<0.001)$ & 0.95 & $<0.0001$ \\
\hline \multicolumn{5}{|c|}{ Calcium removal $\left(\mathrm{kg} \mathrm{Ca} \mathrm{ha}^{-1}\right)$ removal with harvest $\left(\mathrm{Mg} \mathrm{ha}^{-1}\right.$, first and second cut combined) } \\
\hline Jefferson & $1.13(0.5686)$ & $3.86(<0.0001)$ & 0.81 & $<0.0001$ \\
\hline St Lawrence & $-3.95(0.0290)$ & $6.49(<0.0001)$ & 0.97 & $<0.0001$ \\
\hline Columbia & $-9.07(0.0486)$ & $6.76(<0.0001)$ & 0.93 & $<0.0001$ \\
\hline Essex & $-19.77(0.0332)$ & $9.45(<0.0001)$ & 0.84 & $<0.0001$ \\
\hline Cayuga & $-3.78(0.0826)$ & $6.32(<0.0001)$ & 0.95 & $<0.0001$ \\
\hline Tompkins & $-2.58(0.0947)$ & $4.31(<0.0001)$ & 0.97 & $<0.0001$ \\
\hline \multicolumn{5}{|c|}{ Magnesium removal $\left(\mathrm{kg} \mathrm{Mg} \mathrm{ha}^{-1}\right)$ removal with harvest $\left(\mathrm{Mg} \mathrm{ha}^{-1}\right.$, first and second cut combined) } \\
\hline Jefferson & $-0.12(0.9213)$ & $2.05(<0.0001)$ & 0.76 & $<0.0001$ \\
\hline St Lawrence & $-8.18(<0.0001)$ & $5.83(<0.0001)$ & 0.97 & $<0.0001$ \\
\hline Columbia & $-20.98(0.0035)$ & $9.14(<0.0001)$ & 0.92 & $<0.0001$ \\
\hline Essex & $-23.01(0.0258)$ & $6.54(<0.0001)$ & 0.66 & $<0.0001$ \\
\hline Cayuga & $-5.99(0.0041)$ & $4.49(<0.0001)$ & 0.93 & $<0.0001$ \\
\hline Tompkins & $-3.92(0.0132)$ & $3.28(<0.0001)$ & 0.95 & $<0.0001$ \\
\hline
\end{tabular}

See Table 1 for a description of the sites.

in Ca removal (Table 5). Calcium removal rates at the manured site in Columbia County were lower than determined in the swine manured fields in the study in Mississippi possibly due to higher $\mathrm{Ca}$ levels in the calcareous silty clay soils in Mississippi (McLaughlin et al. 2004).

Magnesium removal increased with the addition of $\mathrm{N}$, although increases were not significant in the Jefferson County and Essex County trials (Table 4). Magnesium removal rates were high in the sites in Essex and Columbia County most likely due to a $\mathrm{K}$ deficiency (Essex County) and manure addition (Columbia County). At five sites, DM yield explained more than $75 \%$ of the variability in $\mathrm{Mg}$ removal. The exception was the Essex County site $(66 \%)$, again suggesting a potential K deficiency.

Nutrient removal rates and forage nutrient concentrations were not clearly associated with initial soil fertility levels with the exception of the Essex County trial, where a one time application of $90 \mathrm{~kg} \mathrm{~K}_{2} \mathrm{O}$ ha $^{-1}$ was insufficient to overcome $\mathrm{K}$ deficiency.

\section{Conclusions}

Although site-to-site differences existed, $\mathrm{N}$ application tended to decrease $\mathrm{P}$ and $\mathrm{K}$ concentrations 
and increase $\mathrm{N}, \mathrm{Ca}$ and $\mathrm{Mg}$ concentrations in BMR $\mathrm{S} \times \mathrm{S}$ forage. Increases in yield resulted in highly significant positive relationships between nutrient removal and $\mathrm{N}$ application for all sites except one site that showed a $\mathrm{K}$ deficiency. We conclude that dry matter yields can be used to determine crop nutrient removal but that forages need to be analysed for nutrient content to take site-to-site differences into account. Uptake of all nutrients except $\mathrm{Ca}$ was highest in the manured site. The crop removed large amounts of $\mathrm{K}$ in the manured site. If manure is applied mid-season, forage $\mathrm{K}$ levels are likely too high for feeding of the forage to non-lactating cows.

\section{Acknowledgements}

This research was funded with grants from the Northern New York Agricultural Development Program (NNYADP), the Northeast Region Potash and Phosphate Institute, and Garrison and Townsend Inc. Ammonium sulfate was donated by Honeywell Inc. and seed was supplied by Agriculver. We thank Leon Hatch, Kelly Reardon, and Andy Durow for assistance with harvesting and soil and plant sample processing, and Francoise Vermeylen for help with the statistical analyses.

\section{References}

Beede, D. K., 1996: Cation-anion differences in dairy rations: dealing with high potassium content of alfalfa. In: Proceedings of the 26th National Alfalfa Symposium, East Lansing, MI, 5-6 March 1996, pp. 30-37. Cert. Alfalfa Seed Council, Inc., Davis CA.

Beyaert, R. P., and R. C. Roy, 2005: Influence of nitrogen fertilization on multi-cut forage sorghum-sudangrass yield and nitrogen use. Agron. J. 97, 1493-1501.

Brink, G. E., G. A. Pederson, K. R. Sistani, and T. E. Fairbrother, 2001: Uptake of selected nutrients by temperate grasses and legumes. Agron. J. 93, $887-890$.

Cherney, J. H., D. J. R. Cherney, and T. W. Bruulsema, 1998: Chapter 6. Potassium management. In: J. H. Cherney, and D. J. R. Cherney, eds. Grass for Dairy Cattle, pp. 147-148. CAB Intl, Wallingford, UK.

Cline, M. G., and R. L. Marshall, 1977: Soil of New York Landscapes. Media and Technology Services, Cornell University, Ithaca, NY.

Cornell Cooperative Extension, 2006: Cornell Guide for Integrated Field Crop Management. Media and Technology Services, Cornell University, Ithaca, NY.

Dairy One, 2006: Field Composition Library. Available at: http://www.dairyone.com/Forage/FeedComp/ mainlibrary.asp. Accessed on 4 March 2005.

Daly, K., and A. Casey, 2003: Eutrophication from Agricultural Sources. Environmental Soil Phosphorus
Test (2000-LS-2.1.6-M2). Final report prepared for the Environmental Protection Agency. Teagasc, Johnstown Castle, Wexford, Ireland.

Goff, J. P., and R. L. Horst, 1997: Effects of the addition of potassium or sodium, but not calcium, to prepartum rations on milk fever in dairy cows. J. Dairy Sci. 80, $176-186$.

Greweling, T., 1976: Dry ashing. Cornell Univ. Agric. Exp. Stn. Res. Bull. 6, 1-35.

Ketterings, Q. M., S. D. Klausner, and K. J. Czymmek, 2003: Nitrogen Guidelines for Field Crops in New York. 2nd Release. Dept. Crop Soil Sci. Ext Series E03-16, p. 70.Cornell University, Ithaca, NY.

Ketterings, Q. M., T. F. Kilcer, P. Cerosaletti, and J. H. Cherney, 2004: Phosphorus removal by sorghumsudangrass in Northeastern USA. Online. Forage and Grazinglands doi:10.1094/FG-2004-1015-01-RS.

Ketterings, Q. M., G. Godwin, J. H. Cherney, and T. F. Kilcer, 2005a: Potassium management for brown midrib sorghum $\times$ sudangrass as replacement for corn silage in the Northeastern USA. J. Agron. Plant Sci. 191, 41-46.

Ketterings, Q. M., S. N. Swink, G. Godwin, K. J. Czymmek, and G. L. Abrecht, 2005b: Maize silage yield and quality response to starter phosphorus fertilizer in high phosphorus soils in New York. J. Food Agric. Environ. 3, 237-242.

Kilcer, T. F., Q. M. Ketterings, J. H. Cherney, P. Cerosaletti, and P. Barney, 2005: Optimum stand height for forage brown midrib sorghum $\times$ sudangrass in Northeastern USA. J. Agron. Plant Sci. 191, 35-40.

McIntosh, J. L., 1969: Bray and Morgan soil test extractants modified for testing acid soils from different parent materials. Agron. J. 61, 259-265.

McLaughlin, M. R., T. E. Fairbrother, and D. E. Rowe, 2004: Forage yield and nutrient uptake of warmseason annual grasses in a swine effluent spray field. Agron. J. 96, 1516-1522.

Morgan, M. F., 1941: Chemical soil diagnosis by the universal soil testing system. Conn. Agric. Exp. Stn. Bull. 450, 579-628.

Nelson, D. W., and L. E. Sommers, 1996: Chapter 34. Total carbon, organic carbon, and organic matter. In: D. L. Sparks, (ed.). Methods of Soil Analyses: Part 3: Chemical Methods. SSSA-ASA Book Series (5), pp. 961-1010. SSSA/ASA, Madison, WI, USA.

Northeast Regional Climate Center, 2006: CLIMOD Database. Available at: http://met-www.cit.cornell.edu/nrcc_web_climod.html. Accessed on 4 March 2006.

PPI/PPIC/FAR, 2002: Plant Nutrient Use in North American Agriculture. PPI/PPIC/FAR Tech. Bull. 2002-1. PPI, Norcross, GA, USA.

SAS Institute Inc., 1999: SAS/STAT User's Guide. Release 8.00. SAS Institute Inc., Cary, NC, USA.

U.S. Environmental Protection Agency, 2000: National Water Quality Inventory Report. Available at: http:// www.epa.gov/305b/2000report/. Accessed on 4 March 2006. 